\title{
The effect of protected mealtimes on meal interruptions, feeding assistance, energy and protein intake and plate waste
}

\author{
C. E. Weekes \\ Department of Nutrition \& Dietetics, Guy's \& St Thomas' NHS Foundation Trust, Lambeth Palace Road, \\ London SE1 7EH, UK
}

Protected mealtimes (PM) i.e. periods on a hospital ward when all non-urgent clinical activity stops, have been recommended as a method for improving dietary intake in hospitalised patients. There is however, a lack of data to support this strategy.

Two 5-d surveys were conducted at lunchtimes on one elderly care ward in an acute hospital trust. Survey 1 was conducted prior to introduction of PM and Study 2 was conducted 1 month after introduction of PM. Patients were observed by volunteers (Trust nursing and dietetic staff) prior to and during the lunchtime meal, until their tray was cleared away by the food service assistant. The following parameters were recorded: number of meal interruptions; feeding difficulties; feeding assistance provided to aid meal consumption. Nutritional intake and plate waste were assessed in a random sample of ten meals per day. Each meal was weighed on the ward immediately before the patient was handed the tray and again immediately after the patient had finished (Table). Nutritional analysis was conducted using Microdiet Version 1.1 (University of Salford, UK).

\begin{tabular}{lccc}
\hline & Survey 1 & Survey 2 & $P$ \\
\hline Patient meals observed & 126 & 106 & - \\
Meal interruptions (median (range)) & $0(0-3)$ & $0(0-2)$ & $0.005^{*}$ \\
Feeding difficulties (median (range)) & $1(0-3)$ & $0(0-3)$ & $0.036^{*}$ \\
Feeding assistance provided (median (range)) & $1(0-3)$ & $0(0-3)$ & $0.004^{*}$ \\
Meals weighed & 54 & 56 & \\
Energy intake (kJ) & 1761 (SD 887) & 1577 (SD 833) & $0.268 \dagger$ \\
Protein intake (g) & 17.6 (SD 10) & 15.9 (SD 9.2) & $0.368 \dagger$ \\
Plate waste & $27 \%$ (SD 24) & $32 \%$ (SD 30) & $0.286 \dagger$ \\
\hline
\end{tabular}

$* \chi^{2}$ test; $\dagger$ unpaired $t$ test.

The number of interruptions significantly decreased following PM, mainly the result of fewer visits by the phlebotomist and allied health professionals, and altered timing of drug rounds. Observed feeding difficulties were similar in both surveys i.e. patients unable to cut up their food (about $33 \%$ ) or open packets (about $25 \%$ ) or unable to feed themselves due to physical or cognitive impairment (about $15 \%)$. The number of staff on the ward at lunchtime was comparable in both surveys i.e. three or four nurses and between one and three health care assistants (total seven staff/d), yet a significant decrease in feeding assistance was observed during Survey 2.

In this study PM resulted in fewer meal interruptions. The absence of an increase in feeding assistance however, may at least partially account for the lack of beneficial effects on energy and protein intake or plate waste. The barriers to providing adequate feeding assistance merit further investigation. 